\title{
Ion escape from the high latitude magnetopause: analysis of oxygen and proton dynamics in the presence of magnetic turbulence
}

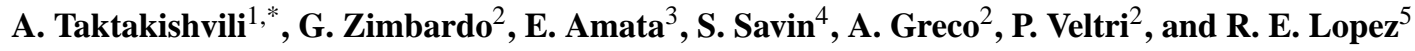 \\ ${ }^{1}$ Center for Plasma Astrophysics, Tbilisi, Georgia \\ ${ }^{2}$ Universitá della Calabria, Cosenza, Italy \\ ${ }^{3}$ Istituto di Fisica dello Spazio Interplanetario, INAF, Roma, Italy \\ ${ }^{4}$ Space Research Institute, Moscow, Russia \\ ${ }^{5}$ Florida Institute of Technology, Melbourne, FL, USA \\ *also at: Goddard Space Flight Center, Greenbelt, MD, USA
}

Received: 9 December 2006 - Revised: 30 May 2007 - Accepted: 13 July 2007 - Published: 29 August 2007

\begin{abstract}
Recent Cluster observations of the vicinity of the high latitude magnetopause indicate the presence of beams of singly charged oxygen ions, which are of ionospheric origin. In this paper we examine the role of magnetic turbulence combined with a dc electric field across the magnetopause in causing the cross field transport of protons and of singly charged oxygen ions, by means of a kinetic test particle simulation. We find that the observed values of magnetosheath turbulence and electric fields can produce a substantial escape of the oxygen ions relative to protons. By varying the magnetic turbulence level in the simulation, we find that the number of $\mathrm{O}^{+}$crossing the magnetopause grows with $\delta B / B_{0}$, and that very few ions can cross the magnetopause for $\delta B / B_{0}=0$. The ion temperature also grows with $\delta B / B_{0}$, showing that magnetic turbulence is effective in thermalizing the kinetic energy gain due to the cross-magnetopause potential drop. We suggest that this mechanism can help to explain Cluster observations of energetic oxygen ions during a high-latitude magnetopause crossing.
\end{abstract}

Keywords. Magnetospheric physics (Magnetopause, cusp, and boundary layers) - Space plasma physics (Numerical simulation studies; Transport processes)

\section{Introduction}

It was suggested by Haerendel et al. (1978) that the magnetospheric regions above the polar cusps would correspond to strong perturbations of the magnetic and velocity fields. In situ measurements by, among the others, the Prognoz 8, In-

Correspondence to: G. Zimbardo

(zimbardo@fis.unical.it) terball, Polar, and Cluster spacecraft have shown that this is indeed the case (Savin et al., 1998, 2002, 2005a; Nykyri et al., 2004, 2006; Sundkvist et al., 2005b). The strong turbulence observed could be due either to magnetic reconnection occurring at the high latitude magnetopause (Scudder et al., 2002), or to instabilities related to the shear flows in the magnetosheath, or to kinetic instabilities at frequencies around the local proton gyrofrequency (Sahraoui, 2006; Nykyri et al., 2006; Zimbardo, 2006). Magnetic turbulence in the cusp region exhibits a power spectrum which often has a double slope, with spectral index around 1.2-1.7 below the proton gyrofrequency, and around 2.5-4 above the proton gyrofrequency (Nykyri et al., 2006). Further, coherent structures like plasma bubbles and Alfvenic vortices are observed close to the cusp region (Sundkvist et al., 2005a; Savin et al., 2005a), with characteristic scales of the order of the proton gyroradius (drift-kinetic Alfven vortices) (Sundkvist et al., 2005a). Bicoherence analysis shows that nonlinear 3-wave interactions are going on in the magnetopause (Savin et al., 2005b). In addition, the polar cusps are often populated with high energy particles, with energies up to a few $\mathrm{MeV}$ (Chen et al., 1998); the origin of these particles is still a matter of debate: the possibility that they are accelerated locally indicates that the cusps are regions of intense energy conversion, and that they can be an ideal laboratory for nonlinear plasma processes (Blecki et al., 2005).

On the other hand, singly charged oxygen ions, of ionospheric origin, are often observed in the magnetosphere (Shelley et al., 1972; Lockwood et al., 1985; Moore et al., 1986; Yau and Andre, 1997; Andre and Yau, 1997; Chappell et al., 2000; Peroomian et al., 2006). Recently, during several crossings of the high latitude magnetopause, Cluster observed $\mathrm{O}^{+}$beams with energies of $10-20 \mathrm{keV}$ (Bogdanova et

Published by Copernicus Publications on behalf of the European Geosciences Union. 
al., 2004; Nilsson et al., 2006). In particular, $\mathrm{O}^{+}$beams were observed during the Cluster magnetopause crossing of 13 February 2001, at around 20:00 (Amata et al., 2006). For this event, close inspection of Cluster CODIF data (not shown herein) reveals that a population of singly charged oxygen ions with energies between $10-30 \mathrm{keV}$ and temperatures corresponding to about $10 \mathrm{keV}$ were registered around the magnetopause, with a corresponding cold $\left(T_{\mathrm{O}^{+}} \sim 600 \mathrm{eV}\right)$ oxygen flow (bulk velocity $V_{\mathrm{O}^{+}} \simeq 140 \mathrm{~km} / \mathrm{s}$ ) at lower altitudes. Often, the temperature transverse to the magnetic field is larger than that parallel to the magnetic field. A quasi dc electric potential drop $\Delta \varphi$ normal to the magnetopause, with $\Delta \varphi$ of the order of $10 \mathrm{kV}$, and a substantial level of magnetic fluctuations with $\delta B / B_{0} \sim 0.4$ with frequencies $\nu=0.01-1 \mathrm{~Hz}$, were observed throughout the magnetopause crossing, from 20:00 UT to 20:02 UT, see Fig. 1. Strong electric fluctuations were observed, too, but concentrated near the periods of strongest potential drop, 20:00 UT and 20:15 UT (with the magnetopause current layer observed at 20:00:58 UT).

The mechanisms by which the $\mathrm{O}^{+}$ions escape from the inner magnetosphere to higher altitudes and are energized are not well understood. The proposed energization mechanisms range from magnetic reconnection either at low or at high latitudes, to resonant heating by electric field fluctuations (Andre and Yau, 1997; Bogdanova et al., 2004), to centrifugal acceleration of the outflowing ions (Nilsson et al., 2006). We point out that understanding the escape mechanism can be instrumental in recognizing the actual energization mechanism. For instance, if $\mathrm{H}^{+}$and $\mathrm{O}^{+}$ions cross the magnetopause locally, the energization mechanism could be local, too. On the other hand, ion energization can also occur at lower altitudes, thanks to a variety of mechanisms like ion resonance with perpendicular electric fields associated with broadband low-frequency waves, leading to ion conics, or field aligned ion acceleration due to the ponderomotive force (Andre and Yau, 1997).

In this paper we present the results of a numerical simulation of oxygen and proton dynamics in the turbulent magnetopause configuration. We develop a simple model of the magnetosphere-magnetosheath transition which mimics some of the main features observed by spacecraft. The main idea is that magnetic turbulence provides ions with the mobility across the magnetopause. In this case they may be accelerated by the quasi-dc potential drop, as well as by other mechanisms. In the numerical simulation, $\mathrm{H}^{+}$and $\mathrm{O}^{+}$ions are injected at one boundary of the simulation box with velocities similar to those observed. A sheared magnetic field with components $B_{y}, B_{z}$ represents the magnetopause and an electric field $E_{x}$ normal to the magnetic field accounts for the observed quasi-dc potential drop. A numerical realization of 3-D magnetic fluctuations was superimposed on the average fields. We perform a parametric study of ion dynamics, changing the values of relevant parameters like the magnetic turbulence level $\delta B / B_{0}$ and the normal electric field $E_{x}$. We find that oxygen transport and heating increase with the increase of turbulence level. Due to different gyroradius, the two species of ions exhibit very different dynamics, with oxygen ions having more chance to penetrate through the magnetopause, be accelerated and gain bulk motion energy effectively, while protons are hardly able to cross the magnetopause, being scattered by the magnetic fluctuations.

In Sect. 2 we present the features of the numerical model. In Sect. 3 we give the numerical results, showing the differences between proton and oxygen behaviour, the dependence of the results on the magnetic turbulence level, and the influence of varying the cross-magnetopause electric field. In Sect. 4 we give the conclusions.

\section{Numerical model}

The numerical model is an upgraded version of the code used by Greco et al. (2003) to study proton transport across the magnetopause. Both protons and oxygen ions are injected into the simulation box, which extends for $L_{x} \equiv L$ in the $x$ (normal to the magnetopause) direction, and is much larger in the y-and z-directions, $L_{y}=L_{z}=30 \mathrm{~L}$. The average magnetic field is given by $B_{z} \simeq B_{0} \tanh (x / \Delta)$, with $\Delta$ the current sheet half-thickness, and $B_{y}=$ const the "shear" magnetic field. We set $\Delta=0.25 L$ (the profile of the magnetic field reversal is slightly modified with respect to Harris sheet, in order to have $B_{z}=B_{0}$ at the boundaries, see Veltri et al., 1998), with the box boundaries at $x= \pm 0.5 L_{x}$. In the present runs, $B_{y}=0.8 B_{0}$, which gives a shear angle slightly larger than $90^{\circ}$. Physical values of the parameters can be obtained by setting $L=2000 \mathrm{~km}$ and $B_{0}=50 \mathrm{nT}$. We note that Amata et al. (2006) report a magnetopause velocity along the normal of $32 \mathrm{~km} / \mathrm{s}$. The region with enhanced turbulence extends for about $90 \mathrm{~s}$ around the magnetopause location, see Fig. 1, so that the thickness of the turbulent region can be estimated as $\sim 2880 \mathrm{~km}$. A simulation box thickness of about $2000 \mathrm{~km}$, centered around the magnetopause crossing, is therefore realistic. The normal electric field $\boldsymbol{E}=-\boldsymbol{V} \times \boldsymbol{B}$ is due to the plasma flow in the magnetosheath parallel to the magnetopause and corresponds in Cluster data to a potential drop of the order of $10 \mathrm{kV}$, with the electric field $E_{x} \simeq 6 \mathrm{mV} / \mathrm{m}$ pointing away from the Earth (Amata et al., 2006). Indeed, the simulatenous presence of a quasi-dc electric field and of magnetic turbulence at the magnetopause is apparent in the considered magnetopause crossing. Therefore, proton and oxygen ions coming from the ionosphere could be accelerated if they could cross the magnetic field.

In the present runs, the electric field is modeled as $E_{x}(x)=E_{0} \exp \left[-\left(x-x_{0}\right)^{2} / x_{0}^{2}\right]$, with $x_{0}=0.25 L$, that is the electric field is centered on the outward side of the magnetopause transition, in agreement with the observations (Amata et al., 2006) and with the fact that the quasi dc $E_{x}$ is due to $\boldsymbol{V} \times \boldsymbol{B}$ in the magnetosheath. For most runs, the peak value is $E_{0}=22.57 \mathrm{mV} / \mathrm{m}$, which corresponds to a potential energy drop $\Delta U=e \Delta \varphi \simeq 17 \mathrm{keV}$ within the simulation box. Another 


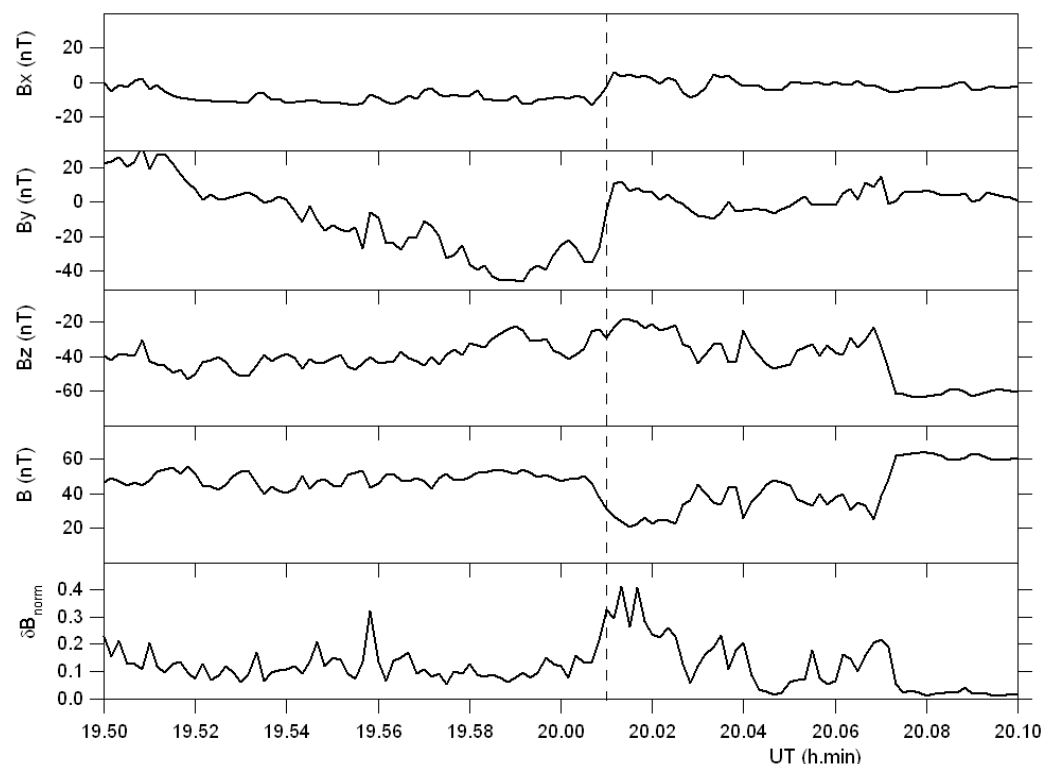

Fig. 1. Magnetic field components measured by the Cluster spacecraft during the 13 February 2001, magnetopause crossing, averaged over $10 \mathrm{~s}$. From top to bottom, the three magnetic field components in the GSM system, the magnetic field magnitude, and the fluctuation level $\delta B$ normalized to the local magnitude are shown. The simulation box of the numerical study corresponds approximately to the measurements going from 20:00:30 UT to 20:01:30 UT.

value, $E_{0}=6 \mathrm{mV} / \mathrm{m}$, corresponding to the measuread electric field, was also used. These choices are due to the fact that to have a realistic potential drop with a realistic electric field would have required a simulation box larger (in $x$ ) than that used, with a corresponding increase in the computational time. The other two components of the electric field are taken equal to zero.

The magnetic fluctuations are modelled, following Greco et al. (2003), as a sum of plane waves

$\delta \mathbf{B}(\mathbf{r})=\sum_{k, \sigma} \delta B(\mathbf{k}) \mathbf{e}_{\sigma}(\mathbf{k}) \exp \left[i\left(\mathbf{k} \cdot \mathbf{r}+\phi_{\mathbf{k}}^{\sigma}\right)\right]$,

where $\mathbf{e}_{\sigma}(\mathbf{k})$ are the polarization unit vectors, and the random phases $\phi_{\mathbf{k}}^{\sigma}$ are chosen so that the fluctuations sum up coherently for $x=0$, so that they are stronger in the center of the simulation box (for more details, see Veltri et al., 1998, and Greco et al., 2003). The Fourier amplitudes $\delta B(\mathbf{k})$ are given by

$\delta B(\mathbf{k})=\frac{C}{\left(k_{x}{ }^{2} l_{x}{ }^{2}+k_{y}{ }^{2} l_{y}{ }^{2}+k_{z}{ }^{2} l_{z}{ }^{2}+1\right)^{\alpha / 4+1 / 2}}$

where $C$ is a normalization constant, which sets the value of the magnetic turbulence level. The wave vectors are chosen on a discrete 3-D grid, with $k_{i}=2 \pi n_{i} / l_{i}, i=x, y, z$, where $n_{i}$ are the harmonic numbers and $l_{i}$ the turbulence correlation lengths. We set $l_{x}=0.05 L_{x}$ and $l_{y}=l_{z}=0.5 L_{x}$ (this also corresponds to the simulation box geometry). Also, $k_{x \min }=2 \pi / L_{x}, k_{x \max }=12 k_{x \min }$, and the spectral index $\alpha=1.5$ is in the range of those observed (Nykyri et al., 2006).
For all runs, 20000 particles were injected at $x=-0.5 \mathrm{~L}$, which represents the magnetospheric side of the simulation box, with random position in the $y z$ plane. The injection velocity corresponds to a thermal velocity of $600 \mathrm{eV}$ and a bulk velocity $50-100 \mathrm{~km} / \mathrm{s}$, making an angle of about $40^{\circ}$ with the average magnetic field direction at the $x=-0.5 \mathrm{~L}$ boundary. Protons were injected with the same initial flow velocity and the same initial temperature. The trajectories of test particles are integrated by means of a 4th 5 th order adaptive step Runge-Kutta routine. A maximum integration step corresponding to the minimum between $0.1 \Omega_{i}^{-1}$ and $1 / 10$ of the time needed to cover the shortest wavelength of the turbulence model was chosen. Here, $\Omega_{i}$ is the ion gyrofrequency. We checked the integration accuracy by requiring that the particle energy be conserved with a relative variation of at most $10^{-6}$, and typically much less. Distribution function moments like density $n$, bulk velocity $\boldsymbol{V}$, and temperature $T$ are computed on a 3-dimensional grid in space (Veltri et al., 1998; Greco et al., 2003).

It was shown by Taktakishvili et al. (2003) and by Greco et al. (2003), that this magnetic turbulence allows the flow of ions across the magnetopause, for magnetic fluctuations levels $\delta B / B_{0} \geq 0.3$. Here, $\delta B$ is the rms value of the fluctuations; we note that in the cusp region values of $\delta B / B_{0}$ of order of $0.5-1$ are not uncommon. During the magnetopause crossing of 13 February 2001, upstream in the magnetosheath and at the magnetopause $\delta B / B_{0} \sim 0.3-0.4$, see Fig. 1. The magnetic fluctuations were strong in the frequency range $10^{-2}-1 \mathrm{~Hz}$ (Nykyri et al., 2006), and we note 

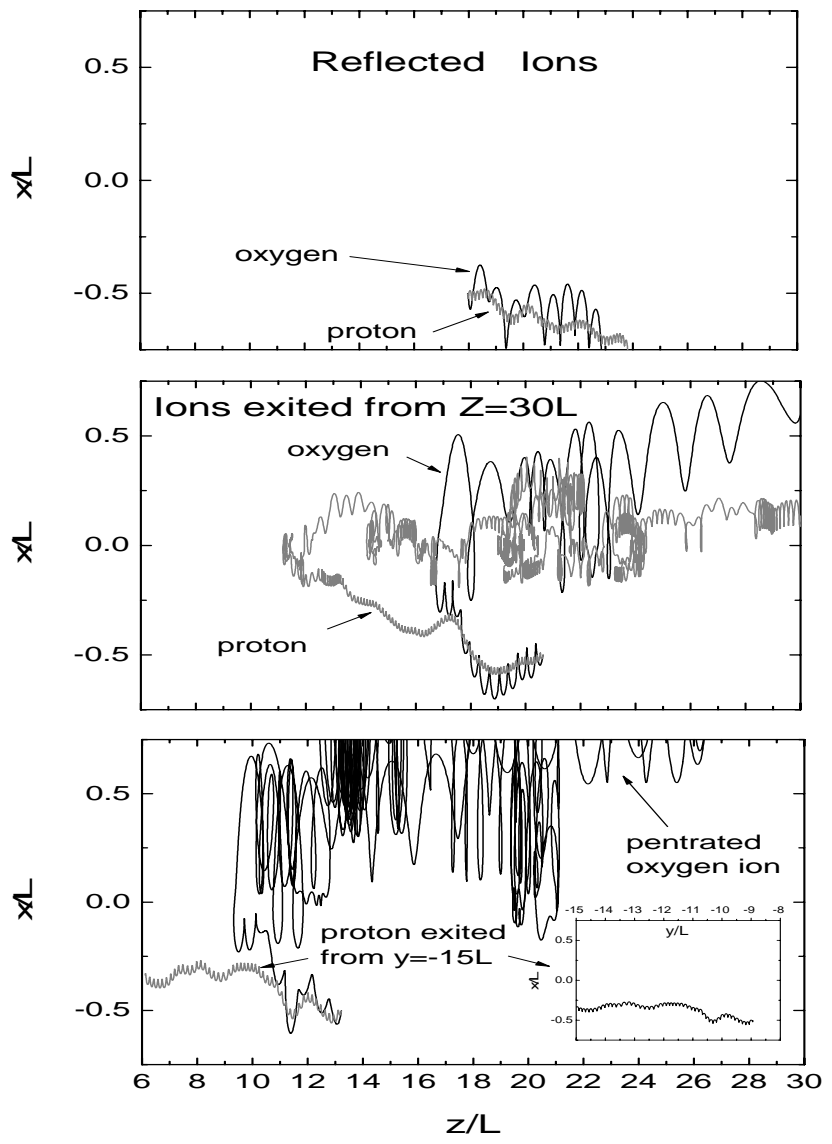

Fig. 2. Sample trajectories of proton and oxygen ions in the simulation box, for $\delta B / B_{0}=0.6$. All the particles are injected at $x=-0.5$ L. Black lines: oxygen. Grey lines: protons. Upper panel: particles exiting from the injection side ("reflected"). Middle panel: particles exiting from the side boundaries. Lower panel: oxygen exiting from the magnetosheath side, proton from the side boundaries.

that the $\mathrm{O}^{+}$gyrofrequency in the $50 \mathrm{nT}$ magnetic field at the magnetopause is $v=4.7 \times 10^{-2} \mathrm{~Hz}$. This resonant interaction corresponds in the simulation to the fact that the oxygen Larmor radius $(\sim 300 \mathrm{~km})$ falls within the range of the turbulence wave lengths in the $\mathrm{x}$-direction $(160 \mathrm{~km}<\lambda<2000 \mathrm{~km})$. Clearly, the proton behaviour can be different because of the smaller Larmor radius.

\section{Simulation results}

In this section we present the results of the simulation, compare oxygen and proton trajectories and present the crossmagnetopause profiles (along the x-direction) of their number density, bulk motion velocity, and kinetic temperature. Then we investigate in more detail the influence of the magnetic turbulence level and of the electric field value on the oxygen transport.

\subsection{Proton vs. oxygen comparison}

In Fig. 2 the $x z$ projections of sample oxygen and proton trajectories are presented. In this figure, oxygen is represented by black solid lines and protons by lighter gray lines. For each of the panels both ion species were launched with the same initial position, i.e., the same randomly chosen y and $\mathrm{z}$ coordinates at the magnetospheric border of the simulation box, $x=-0.5 L$ (bottom section of each panel). The upper part of each panel, $x \geq 0.5 \mathrm{~L}$, represents the magnetosheath part of the simulation box. The upper panel of Fig. 2 shows reflected ions, that is, ions which exit the simulation box from the same side as the injection one. The middle panel shows particles that exit from the flank of the simulation box: both proton and oxygen exit from the $z$ border at $z=30 \mathrm{~L}$. Note that since the initial temperatures of the particles are equal, the proton gyroradius is 4 (square root of mass ratio) times smaller than the oxygen gyroradius. For both ion species, the magnetic turbulence causes a strong deviation from the idealized helical trajectories in simple magnetic field geometries. The protons have a smaller gyroradius and thus are tied more strongly to the magnetic field structure, so that they exhibit a much more stochastic trajectory. Indeed, it can be shown that for large Larmor radii, part of the magnetic fluctuations are averaged along the gyroorbit (Zimbardo, 2005; Pommois et al., 2007). On the other hand, the $\mathrm{O}^{+}$acceleration by the electric field from the middle $(x \sim 0)$ of the simulation box is rather obvious. Both ion species spend most of the time in the central part of the box $x \sim 0$ before exiting, exhibiting a rather prominent average electric drift motion $(\boldsymbol{E} \times \boldsymbol{B})_{z}$ in the positive z-direction in the upper section $(x>0)$, which is the reason of their exit from the simulation box at $z=30 \mathrm{~L}$. The bottom panel of Fig. 2 shows a different behavior of the oxygen and proton ions. The oxygen is strongly accelerated in the initial phase in the cross magnetopause direction by the electric field, and after bouncing close to the region of the strong border magnetic field $x=0.5 \mathrm{~L}$, finally penetrates into the magnetosheath. On the contrary, proton remains all the time close to magnetosphere boundary and finally exits from the simulation box border in the y-direction, $y=15 L$ (see the inset in this panel, showing the projection of the proton trajectory on the $x y$ plane). Note that $E_{x}$ is exponentially small for $x<0$, so that the $(\boldsymbol{E} \times \boldsymbol{B})$ velocity is negligible. These trajectories are most interesting, since they demonstrate the fact that oxygen ions, having larger gyroradius, have more chances to cross the magnetopause and be accelerated by the electric field than protons.

This behaviour is confirmed by Fig. 3, which shows the density, normalized to the injection value at $x=-0.5 L$, as a function of $x$ for the level of fluctuations $\delta B / B_{0}=0.6$. In this and the following figures, the plotted quantities are averaged over the $y$ - and $\mathrm{z}$-directions. It can be seen that a larger number of oxygen ions are able to cross the magnetopause, while proton density drops dramatically for the rightmost, magnetosheath side of the simulation box, $x>0$. In spite of 


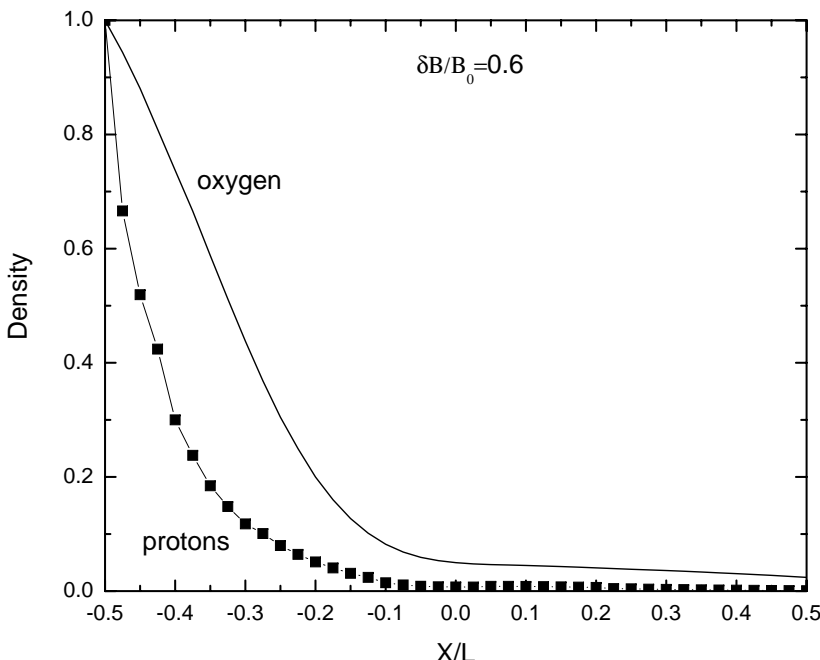

Fig. 3. Density profiles in the simulation box for proton and oxygen ions. Dimensionless units.

the substantial level of magnetic turbulence, very few protons are able to cross the magnetopause.

Figure 4 shows the z-component $V_{\mathrm{bz}}$ of oxygen and proton bulk motion, for the level of fluctuations $\delta B / B_{0}=0.6$. The bulk velocity $V_{\mathrm{bz}}$ is increasing with $x$, because of the acceleration due to $E_{x}$ for both species. Protons appear to be faster, but oxygen ions gain bulk energy more efficiently, due to their larger mass. The behaviour of bulk energy can be inferred from Fig. 4, where it is seen that the oxygen velocity is about one half of the proton velocity, at the right boundary of the simulation box. Due to the mass ratio, this implies that the oxygen bulk energy is about 4 times the proton bulk energy (a minor contribution comes from $V_{\text {by }}$, not shown). This means that in the presence of turbulence ions can cross the magnetopause, but those ions which have larger gyroradius cross quickly and easily, so that they gain an increase of bulk kinetic energy.

Figure 5 shows the temperature growth with $x$ for both particles, normalized to the potential energy drop $\Delta U$. One can see that magnetic fluctuations are an active thermalizing agent. These profiles show more efficient proton heating due to the fact that the fluctuations scatter protons more strongly, causing them a wider spread in particle velocity, which corresponds to an increase in temperature. Conversely, for oxygen ions a larger share of energy goes into bulk kinetic energy rather than thermal energy.

These results show that the turbulent magnetopause exhibits a selective permeability for ions with different masses, allowing heavier oxygen ions to exit from the magnetosphere to the magnetosheath, and be accelerated and heated by the electric field. For protons, on the contrary, the magnetopause appears to be a much more impermeable obstacle, since their smaller gyroradius does not allow them to move across the turbulent magnetopause efficiently.

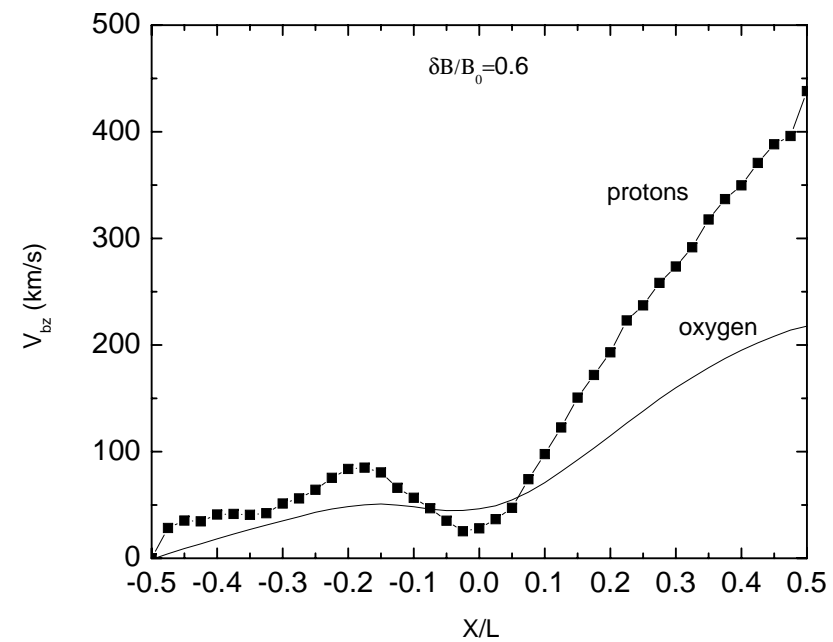

Fig. 4. Bulk velocity along $z$ in the simulation box for proton and oxygen ions.

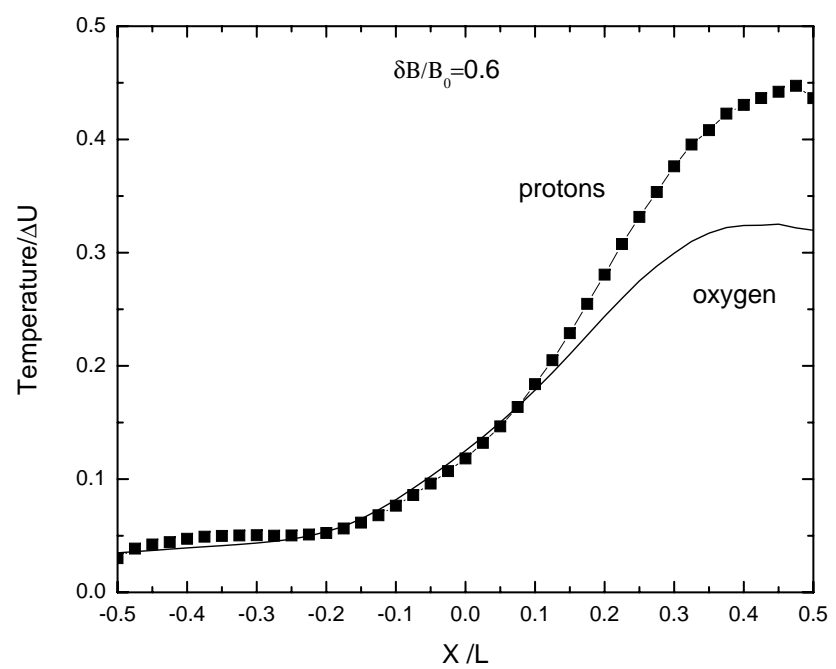

Fig. 5. Temperature profiles for proton and oxygen ions. Dimensionless units.

\subsection{Influence of the turbulence level $\delta B / B_{0}$}

We made a number of runs with oxygen ions only, in order to assess the influence of $\delta B / B_{0}$ and of $E_{0}$ on the transport properties. Figure 6 shows the density profile for $\mathrm{O}^{+}$, for turbulence levels varying from $\delta B / B_{0}=0$ to $0.3,0.6$, and 1.0 , i.e., in the range of those observed at the high latitude magnetopause. While the left side (the injection side) of the density profiles reflects the ion penetration due to the initial Larmor radii (which have an approximately Gaussian distribution), the right side depends on the turbulence level, which is the basic ingredient which allows cross field transport. It can be seen that the height of the density profile increases with the 


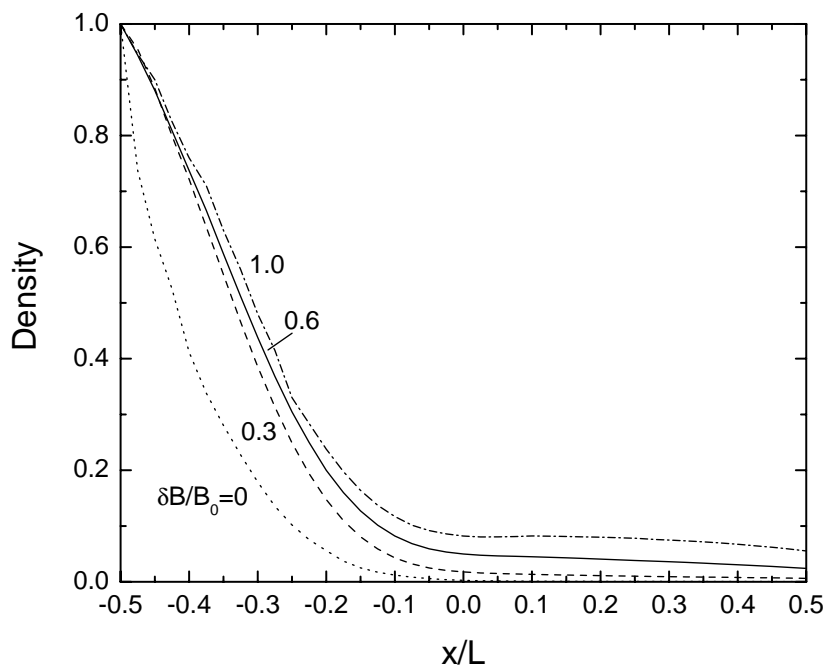

Fig. 6. Oxygen density profile for different turbulence levels (as indicated). Dimensionless units.

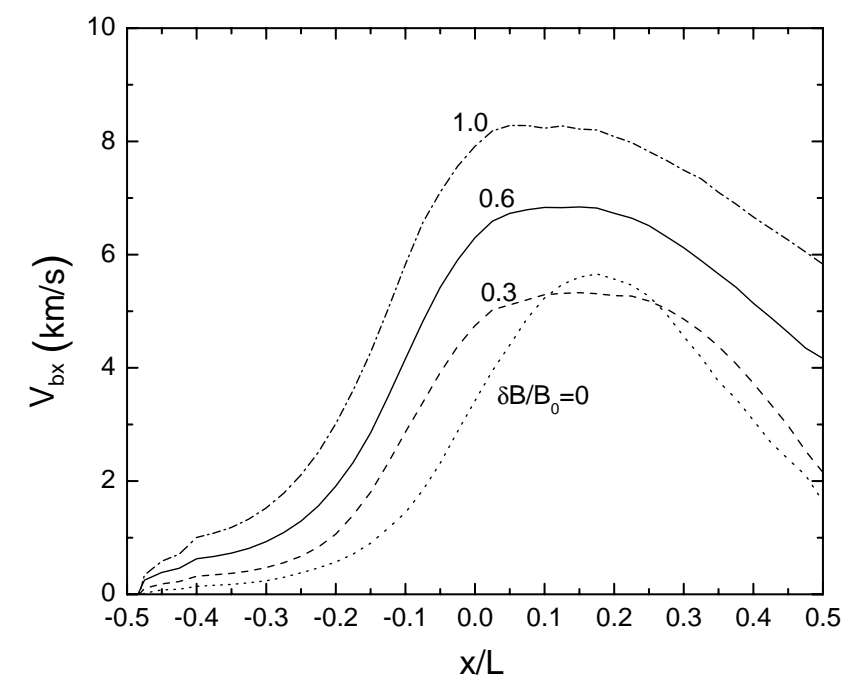

Fig. 7. Oxygen bulk velocity along $x$ for different turbulence levels (as indicated).

turbulence level, in agreement with the results of Greco et al. (2003). Also note that, in practice, almost no oxygen ion is able to cross the current layer for $\delta B / B_{0}=0$.

Figure 7 shows the behaviour of the $x$ component of bulk velocity, $V_{\mathrm{bx}}$. Such a velocity increases with $\delta B / B_{0}$, and corresponds to the flux of ions from the injection (magnetospheric) side at $x=-0.5 \mathrm{~L}$ to the magnetosheath side at $x=0.5 \mathrm{~L}$, with the asymmetry from the negative to positive values of $x$ due to the fact that the electric field is centered at $x_{0}=0.25 \mathrm{~L}$. We point out that the values of the velocity are very small, a few $\mathrm{km} / \mathrm{s}$, which is much smaller than the particle velocities. This means that, in spite of the large gyroradius, $\mathrm{O}^{+}$motion occurs in a diffusive way.

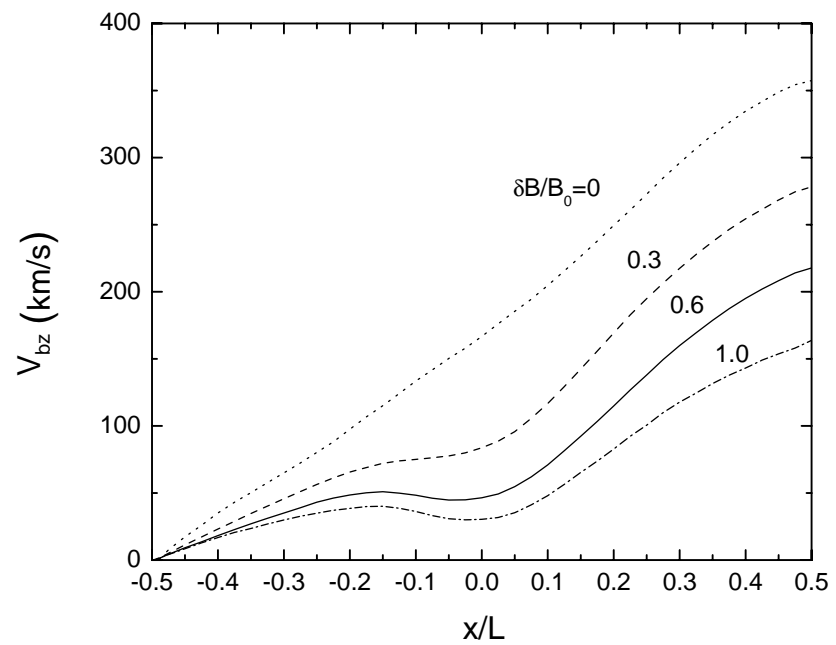

Fig. 8. Oxygen bulk velocity along $z$ for different turbulence levels (as indicated).

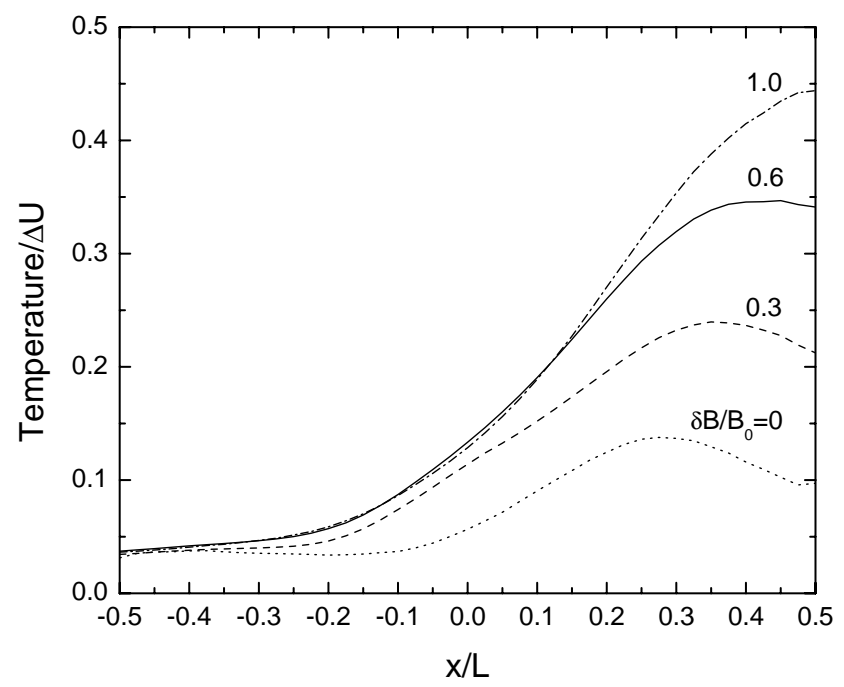

Fig. 9. Oxygen temperature profiles for different turbulence levels. Dimensionless units.

Figure 8 shows the $z$ component of bulk velocity, $V_{\mathrm{bz}}$. It can be seen that it grows from negative to positive values of $x$, and that $V_{\mathrm{bz}}$ is the larger, the smaller $\delta B / B_{0}$. For small $\delta B / B_{0}$, values of $V_{\mathrm{bz}}$ of the order of $300-400 \mathrm{~km} / \mathrm{s}$ are obtained; these large values are due to the z-component of the $\boldsymbol{E} \times \boldsymbol{B}$ drift, given by $V_{\mathrm{Ez}}=E_{x} B_{y} / B^{2}$. Since $B_{y}=$ const, the value of the drift velocity depends mostly on $E_{x}$, which is strongest in the magnetosheath side, and with some modulation by $B^{2}$. We also notice that the obtained values of $V_{\mathrm{bz}}$ are larger than those observed because, in the present runs, $E_{x}$ is larger than that observed, too. On the other hand, when the turbulence level is increased, the particle trajectories are 


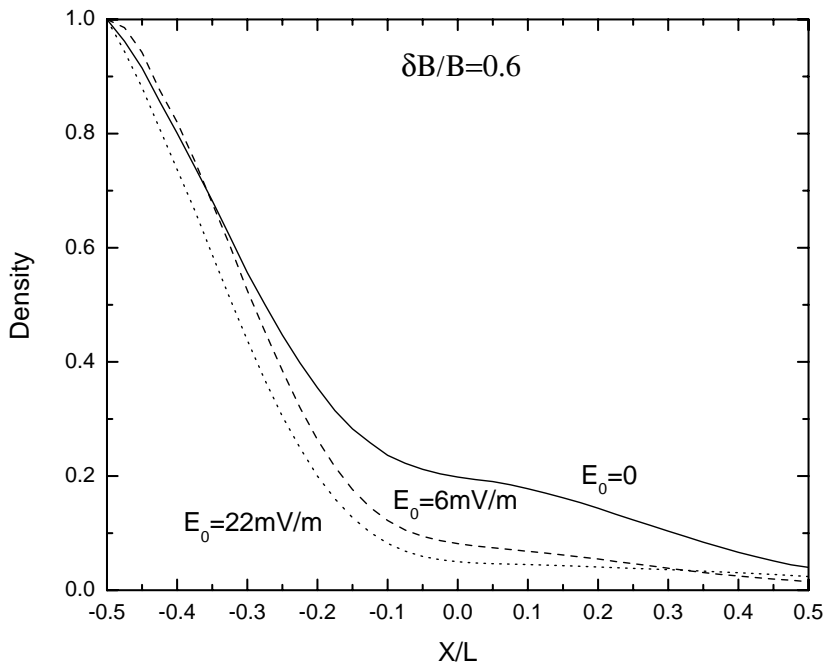

Fig. 10. Oxygen density profiles for different values of the electric field (as indicated). Dimensionless units.

disturbed, and important deflections are caused by $\delta \mathbf{B}$, see Fig. 2, so that the $\boldsymbol{E} \times \boldsymbol{B}$ motion is progressively blurred out, and $V_{\mathrm{bz}}$ decreases. A somewhat similar behaviour is found for $V_{\text {by }}$ (not shown). Note that for large $\delta B / B_{0}$, a smaller fraction of the potential energy due to $E_{x}$ is converted to bulk motion.

Figure 9 shows the corresponding temperature profiles. It can be seen that the temperature increases from the magnetospheric to the magnetosheath sides, and that the larger $\delta B / B_{0}$, the larger the temperature. For the kinetic energy of bulk motion, the opposite trend with $\delta B / B_{0}$ is found (not shown). This confirms that magnetic turbulence plays an important role in thermalizing the kinetic energy obtained by crossing the electric potential drop. As shown by Fig. 5, such a thermalization is more effective for $\mathrm{H}^{+}$than for $\mathrm{O}^{+}$.

\subsection{Influence of the electric field strength $E_{0}$}

We explored the influence of the steady electric field intensity on the transport and energization properties of $\mathrm{O}^{+}$, changing the value of the peak electric field from $E_{0}=0$ to $6 \mathrm{mV} / \mathrm{m}$ and to $22 \mathrm{mV} / \mathrm{m}$, while keeping the magnetic turbulence level to $\delta B / B_{0}=0.6$. Figure 10 shows the oxygen density profile for different electric fields: it can be seen that the density decreases with the increase of $E_{0}$, contrary to naive expectations. We consider that this is due to the influence of the $\boldsymbol{E} \times \boldsymbol{B}$ drift, which grows with $E_{0}$ and which causes the ions to move fast in the $y$ - and z-directions. Indeed, in a stationary one dimensional configuration, the continuity equation requires the flux to be constant, so that the larger the velocity, the smaller the density. Accordingly, the density at around $x=0$ is maximum for $E_{0}=0$, as there is no electric drift and ions lazily spend time in the center of the simulation box,

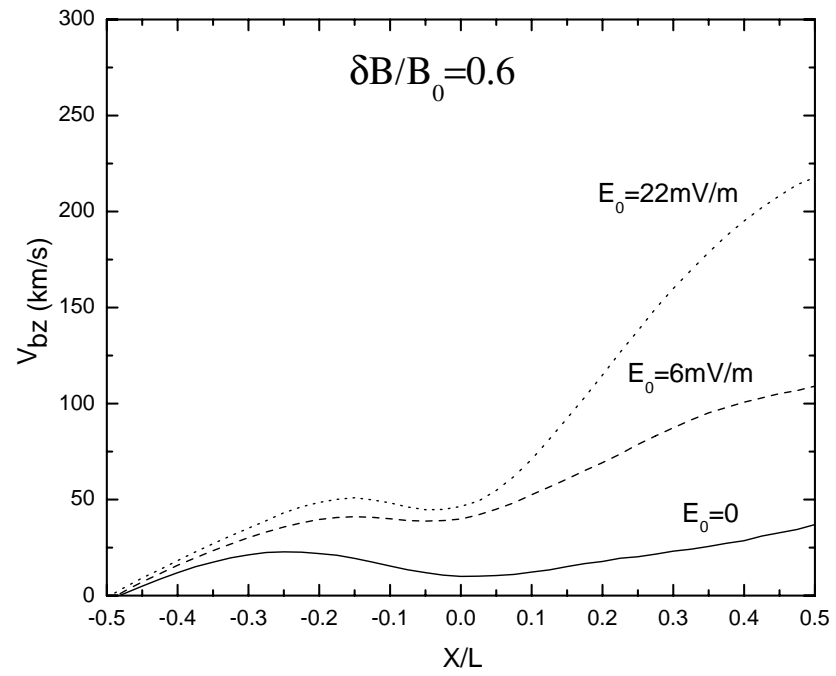

Fig. 11. Oxygen bulk velocity along $z$ for different values of the electric field.

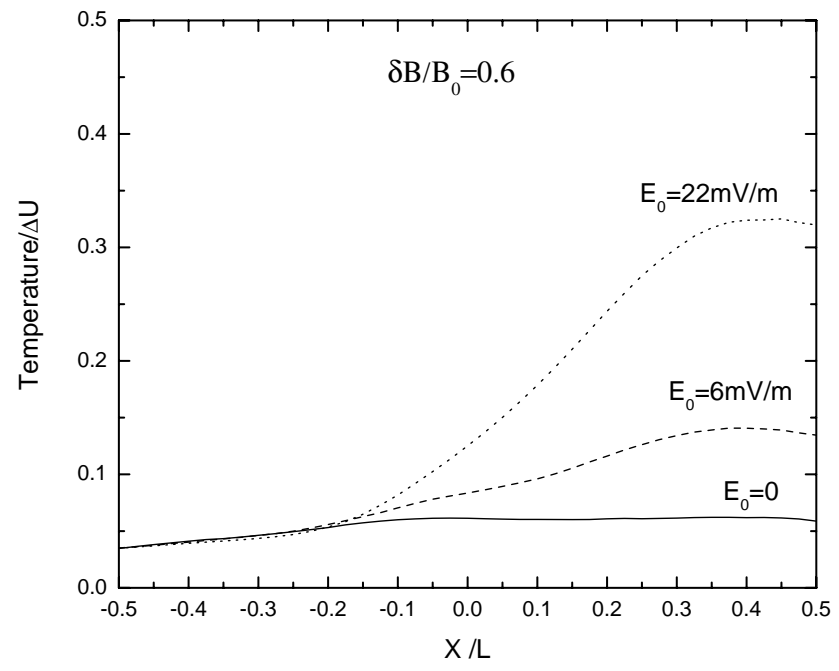

Fig. 12. Oxygen temperature profiles for different values of the electric field. Dimensionless units.

finding their stochastic pathway through the distorted magnetic field. The influence of $E_{0}$ is clearly shown in Fig. 11, where the bulk velocity $V_{\mathrm{bz}}$ is reported, and it is seen that $V_{\text {bz }}$ steadily increases with $E_{0}$, in spite of the relevant turbulence level $\delta B / B_{0}=0.6$. Finally, in Fig. 12 we show the temperature profile for different values of $E_{0}$. All temperatures are normalized to the largest potential energy drop $\Delta U$ corresponding to $E_{0}=22 \mathrm{mV} / \mathrm{m}$. As expected, the stronger $E_{0}$, the larger the temperature increase due to the crossing of the potential drop. For $E=0$, there is no temperature growth, apart from a very small increase which can be related to velocity filtration. 


\section{Conclusions}

We propose that the magnetic fluctuations can be effective in allowing particles to migrate across the magnetopause current layer. We performed a comparative numerical analysis of oxygen and proton ion dynamics in the Earth's magnetopause in the presence of magnetic turbulence and cross magnetopause electric field. The fluctuations and the finite Larmor radius effect allow ions to jump from one magnetic surface to another, gradually being displaced in the cross layer $(x)$ direction. The simulation clearly demonstrates how the mass (Larmor radius) difference between the two species results in a substantial dissimilarity in particle dynamics and, consequently, in difference of the cross-magnetopause profiles of the distribution function moments, such as density, bulk velocity, and temperature. It appears that heavier oxygen ions are more likely to exit from the magnetosphere to the magnetosheath and be orderly accelerated by the electric field. Protons, due to their smaller gyro radius, are hardly able to cross the magnetopause, being effectively scattered by the magnetic fluctuations and heated. The reported results of a selective permeability of the turbulent magnetopause could be used to explain the Cluster observation of energetic oxygen ions on 13 February 2001, and show that oxygen ions can escape locally from the magnetopause. Analysis of Cluster data in the magnetotail, too, shows that the proton and singly charged oxygen dynamics can be very different (Kistler et al., 2005), with the $\mathrm{O}^{+}$ions exhibiting a nonadiabatic behaviour.

By varying the magnetic turbulence level in the simulation, we have shown that the number of $\mathrm{O}^{+}$crossing the magnetopause grows with $\delta B / B_{0}$, and that very few ions can cross the magnetopause for $\delta B / B_{0}=0$. The ion temperature also grows with $\delta B / B_{0}$, showing that magnetic turbulence is effective in thermalizing the kinetic energy gain due to the cross-magnetopause potential drop. It appears that this potential drop may give a contribution to the oxygen energization, although additional mechanisms have to be considered in order to reach the $10-20 \mathrm{keV}$ of the observed $\mathrm{O}^{+}$beams (e.g., Andre and Yau, 1997; Yau and Andre, 1997; Chappell et al., 2000; Bogdanova et al., 2004; Nilsson et al., 2006). On the other hand, populations of very energetic ions with energies from $10 \mathrm{keV}$ up to a few $\mathrm{MeV}$ are often observed in the cusp regions as well (Chen et al., 1998; Chen and Fritz, 2001; Fritz et al., 2003). The acceleration mechanisms for these particles are not yet clear (Chen al al., 1998; Fritz et al., 2000), although electromagnetic turbulence is likely to play an important role. This issue will be considered in a future paper.

Acknowledgements. This research was partially supported by grants INTAS-03-50-4872, INTAS 05-100008-8050, RFFR 06-0217256, and INTAS 06-1000017-8943. Further support was given by the Italian Istituto Nazionale di Astrofisica (INAF), and by the Agenzia Spaziale Italiana (ASI).
Topical Editor I. A. Daglis thanks D. Sundqvist and another anonymous referee for their help in evaluating this paper.

\section{References}

Amata, E., Savin, S., Andre, M., et al.: Experimental study of nonlinear interaction of plasma flow with charged thin current sheets: 1. Boundary structure and motion, Nonlin. Processes Geophys., 13, 1-12, 2006, http://www.nonlin-processes-geophys.net/13/1/2006/.

Andre, M. and Yau, A.: Theories and observations of ion energization and outflow in the high latitude magnetosphere, Space Sci. Rev., 80, 27-48, 1997.

Blecki, J., Wronovski, R., Savin, S., et al.: Low-frequency plasma waves in the outer polar cusp: A review of observations from PROGNOZ 8, INTERBALL 1, MAGION 4, and CLUSTER, Surv. Geophys., 26, 177-191, 2005.

Bogdanova, Y. V., Klecker, B., Paschmann, G., Kistler, L. M., et al.: Investigation of the source region of ionospheric oxygen outflow in the cleft/cusp using multi-spacecraft observations by CIS onboard Cluster, Adv. Space Res., 34, 2459-2464, 2004.

Chappell, C. R., Giles, B. L., Moore, T. E., Delcourt, D. C., Craven, P. D., and Chandler, M. O.: The adequacy of the ionospheric source in supplying magnetospheric plasma, J. Atmos. Sol. Terr. Phys., 62, 421-436, 2000.

Chen, J. and Fritz, T. A.: Correlation of cusp MeV helium with turbulent ULF power spectra and its implications, Geophys. Res. Lett., 25, 4113-4116, 1998.

Chen, J. and Fritz, T. A.: Energetic oxygen ions of ionospheric origin observed in the cusp, Geophys. Res. Lett., 28, 1459-1462, 2001.

Chen, J., Fritz, T. A., Sheldon, R. B., et al.: A new, temporarily confined population in polar cap during the August 27, 1996 geomagnetic field distortion period, Geophys. Res. Lett., 24, 14471450, 1997.

Chen, J., Fritz, T. A., Sheldon, R. B., et al.: Cusp energetic particle events: Implications for a major acceleration region of the magnetosphere, J. Geophys. Res., 103, 69-78, 1998.

Fritz, T. A.: The role of the cusp as a source for magnetospheric particles: A new paradigm, in: ESA Special Publication of the Proceedings of the Cluster II Workshop on Multiscale/Multipoint Plasma Measurements held at Imperial College, London, 22-24 September 1999, ESA SP-499, 2000.

Fritz, T. A., Chen, J. and Siscoe, G.: Energetic ions, large diamagnetic cavities, and Chapman-Ferraro cusp, J. Geophys. Res., 108, 1028, doi:10.1029/2002JA009476, 2003.

Greco, A., Taktakishvili, A. L., Zimbardo, G., et al.: Ion transport and Levy random walk across the magnetopause in the presence of magnetic turbulence, J. Geophys. Res., 108, 1395, doi:10.1029/2003JA010087, 2003.

Haerendel, G., Paschmann, G., Sckopke, N., and Rosenbauer, H.: The frontside boundary layer of the magnetosphere and the problem of reconnection, J. Geophys. Res., 83, 3195-3216, 1978.

Kistler, L. M., Mouikis, C., Möbius, E., et al.: Contribution of nonadiabatic ions to the cross-tail current in an $\mathrm{O}^{+}$dominated thin current sheet, J. Geophys. Res., 110, A06213, doi:10.1029/2004JA010653, 2005.

Lockwood, M., Waite Jr., J. H., Moore, T. E., Johnson, J. F. E., and Chappell, R.: A new source of suprathermal $\mathrm{O}+$ ions near the 
dayside polar cap boundary, J. Geophys. Res., 90, 4099-4116, 1985.

Moore, T. E., Lockwood, M., Chandler, M. O., Waite Jr., J. H., Chappell, C. R., et al.: Upwelling O+ ion source characteristics, J. Geophys. Res., 91, 7019-7031, 1986.

Nilsson, H., Waara, M., Arvelius, S., Marghitu, O., et al.: Characteristics of high altitude oxygen ion energization and outflow as observed by Cluster: a statistical study, Ann. Geophys., 24, 1099-1112, 2006, http://www.ann-geophys.net/24/1099/2006/.

Nykyri, K., Cargill, P. J., Lucek, E., Horbury, T. S., et al.: Cluster observations of magnetic field fluctuations in the high-latitude cusp, Ann. Geophys., 22, 2413-2418, 2004, http://www.ann-geophys.net/22/2413/2004/.

Nykyri, K., Grison, B., Cargill, P. J., Lavraud, B., Lucek, E., et al.: Origin of the turbulent spectra in the high-latitude cusp: Cluster spacecraft observations, Ann. Geophys., 24, 1057-1075, 2006, http://www.ann-geophys.net/24/1057/2006/.

Peroomian, V., El-Alaoui, M., Abdalla,, M. A., and Zelenyi, L. M.: Dynamics of ionospheric $\mathrm{O}^{+}$ions in the magnetosphere during the 24-25 September 1998 magnetic storm, J. Geophys. Res., 111, A12203, doi:10.1029/2006JA011790, 2006.

Pommois, P., Zimbardo, G., and Veltri, P.: Anomalous, non Gaussian transport of charged particles in anisotropic magnetic turbulence, Phys. Plasmas, 14, 012311-012322, doi:10.1063/1.2434795, 2007.

Sahraoui, F., Belmont, G., Rezeau, L., Cornilleau-Wehrlin, N., Pincon, J. L., and Balogh, A.: Anisotropic turbulent spectra in the terrestrial magnetosheath as seen by the Cluster spacecraft, Phys. Rev. Lett. 96, 075002, 2006.

Savin, S. P., Romanov, S. A., Fedorov, A. O., Zelenyi, L. M., Klimov, S. I., et al.: The cusp/magnetosheath interface on May 29, 1996: Interball-1 and Polar observations, Geophys. Res. Lett., 25, 2963-2966, 1998.

Savin, S. P., Zelenyi, L. M., Maynard, N. C., Sandhal, I., et al.: Multi-spacecraft tracing of turbulent boundary layer, Adv. Space Res., 30, 2821-2830, doi:10.1016/S0273-1177(02)804227, 2002.

Savin, S., Zelenyi, L. M., Amata, E., et al.: Dynamical interaction of plasma flow with the hot boundary layer of Geomagnetic trap, JETP Letters., 79, 368-371, 2004.

Savin, S., Skalsky, A., Zelenyi, L. M., et al.: Magnetosheath interaction with the high latitude magnetopause, Surv. Geophys. 26, 95-133, 2005a.

Savin, S., Zelenyi, L., Amata, E., Buechner, J., Blecki, J., Greco, A., Klimov, S., Lopez, R. E., Nikutowski, B., Panov, E., Pickett, J., Rauch, J. L., Romanov, S., Song, P., Skalsky, A., Smirnov, V., Taktakishvili, A., Veltri, P., and Zimbardo, G.: Magnetosheath interaction with high latitude magnetopause: Dynamic flow chaotization, Planet. Space Sci., 53, 133-140, 2005b.
Scudder, J. D., Mozer, F. S., Maynard, N. C., and Russell, C. T.: Fingerprints of collisionless reconnection at the separator, I, Ambipolar-Hall signatures, J. Geophys. Res., 107, 1294, doi:10.1029/2001JA000126, 2002.

Shelley, E. G., Sharp, R. D., and Johnson, R. G.: Satellite observations of energetic heavy ions during a geomagnetic storm, J. Geophys. Res., 77, 6104-6112, 1972.

Sundkvist, D., Krasnoselskikh, V., Shukla, P. K., et al.: In situ multi-satellite detection of coherent vortices as a manifestation of Alfvenic turbulence, Nature, 436, 825, doi:10.1038/nature03931, 2005a.

Sundkvist, D., Vaivads, A., André, M., et al.: Multi-spacecraft determination of wave characteristics near the proton gyrofrequency in high-altitude cusp, Ann. Geophys., 23, 983-995, 2005b.

Taktakishvili, A., Greco, A., Zimbardo, G., Veltri, P., and Zelenyi, L.: Ion penetration into the magnetosphere through the turbulent magnetopause, Adv. Space Res., 31, 1437-1442, 2003.

Veltri, P., Zimbardo, G., Taktakishvili, A. L., and Zelenyi, L. M.: Effect of magnetic turbulence on the ion dynamics in the distant magnetotail, J. Geophys. Res., 103, 14 897-14 916, 1998.

Yau, A. and Andre, M.: Sources of ion outflow in the high latitude ionosphere, Space Sci. Rev., 80, 1-25, 1997.

Zimbardo, G.: Anomalous particle diffusion and Levy random walk of magnetic field lines in three-dimensional solar wind turbulence, Plasma Phys. Control. Fusion, 47, B755, doi:10.1088/0741-3335/47/12B/S57, 2005.

Zimbardo, G.: Magnetic turbulence in space plasmas: in and around the Earth's magnetosphere, Plasma Phys. Control. Fusion, 48, B295, doi:10.1088/0741-3335/48/12B/S28, 2006.

Zimbardo, G., Veltri, P., Basile, G., and Principato, S.: Anomalous diffusion and Lévy random walk of magnetic field lines in three dimensional turbulence, Phys. Plasmas, 2, 2653-2663, 1995.

Zimbardo, G., Greco, A., Veltri, P., Taktakishvili, A. L., Milovanov, A. V., and Zelenyi, L. M.: Ion dynamics in the pre-substorm phase: influence of magnetic turbulence and of the normal component of the magnetic field, in: Proceedings of the Fifth International Conference on Substorms, St. Petersburg, 16-20 May, ESA SP-443, 225-228, 2000a.

Zimbardo, G., Veltri, P., and Pommois, P.: Anomalous, quasilinear, and percolative regimes for magnetic-field-line transport in axially symmetric turbulence, Phys. Rev. E, 61, 1940-1948, 2000b. 